\title{
Water and Ice Adhesion to Solid Surfaces: Common and Specific, the Impact of Temperature and Surface Wettability
}

\author{
Kirill A. Emelyanenko ${ }^{\mathbb{D}}$, Alexandre M. Emelyanenko ${ }^{\mathbb{D}}$ and Ludmila B. Boinovich * \\ A. N. Frumkin Institute of Physical Chemistry and Electrochemistry, Leninsky prospect 31 bldg. 4, \\ 119071 Moscow, Russia; emelyanenko.kirill@gmail.com (K.A.E.); 9554625@mail.ru (A.M.E.) \\ * Correspondence: boinovich@mail.ru; Tel.: +7-495-955-4625
}

Received: 28 May 2020; Accepted: 2 July 2020; Published: 5 July 2020

\begin{abstract}
Ice adhesion plays a crucial role in the performance of materials under outdoor conditions, where the mitigation of snow and ice accumulation or spontaneous shedding of solid water precipitations are highly desirable. In this brief review we compare the adhesion of water and ice to different surfaces and consider the mechanisms of ice adhesion to solids basing on the surface forces analysis. The role of a premelted or quasi-liquid layer (QLL) in the ice adhesion is discussed with the emphasis on superhydrophobic surfaces, and the temperature dependence of ice adhesion strength is considered with an account of QLL. We also very briefly mention some recent methods for the measurement of ice adhesion strength to the icephobic engineering materials outlining the problems which remain to be experimentally solved.
\end{abstract}

Keywords: anti-icing surfaces; work of adhesion; quasi-liquid layer; adhesion strength; superhydrophobicity; icephobic surfaces; temperature dependence; surface forces; wettability; ice adhesion

\section{Introduction}

Adhesion, in the most general sense, is understood as resistance to the breach of the contact of two bodies along the interface. Therefore, in essence, adhesive phenomena are controlled by surface forces acting near the interface. The role of adhesive phenomena in technology and in daily life cannot be overestimated given a variety of applications where they are used. At the same time, for many cases high adhesion is quite harmful. In particular, for products operating in open atmospheric conditions, the adhesion of liquid and solid water precipitations to constructions leads to numerous negative consequences, such as corrosion or ice/snow accretion. Here and below we will consider solid and liquid water precipitations in a broad sense, keeping in mind that in open atmospheric conditions such precipitations represent not pure water but the aqueous solutions with varied $\mathrm{pH}$ and ionic composition. In the aerospace industry, ice adhesion to aircraft structures results in multibillion-dollar industry costs and, in some cases, to human casualties.

The phenomena of adhesion of liquids are the simplest for the analysis since in this case the process of contact failure and the formation of new interfaces are usually thermodynamically reversible. The adhesion of a liquid to the substrate is characterized qualitatively by the contact angle as a measure of intermolecular interactions. For the quantitative characterization of the adhesion, the work of adhesion, calculated by the Young-Dupre equation, is typically used [1-3]. The work of adhesion per unit of area for aqueous medium to any solid at room temperatures is typically quite low and varies from $0.15 \mathrm{~J} / \mathrm{m}^{2}$ to zero. The lowest values correspond to superhydrophobic surfaces due to their water repelling properties characterized by the contact angles close to $180^{\circ}$. 
Considering the spontaneous removal of a separate liquid droplet under the gravitational field or wind, it is the wetting hysteresis that determines the force preventing the movement of a droplet along the surface. The lower the hysteresis, the more efficiently the drops are removed from a solid surface under wind force or air stream pressure $[4,5]$.

One more way of characterization of solid/liquid adhesion, which is intensively developing during recent years is based on the measurement of the maximum adhesion and pull-off forces between the solid surface and the suspended droplet using a microelectronic balance system $[5,6]$.

The characterization of the failure of the contact between the two solid bodies is much more complex. Similar to liquid/solid contact, the surface forces play a determining role here, too. Depending on the type of intermolecular interactions in the adjacent phases, either physical interactions like the dispersion and electrostatic forces or chemical interactions manifesting through hydrogen bonding, heteropolar or homeopolar bonding, come into play [7]. The breaking of the contact between two bodies is crucially affected by the interface roughness. Besides, adhesive contact separation is accompanied by the significant dissipative losses of the energy, related to local heating and elastic or viscoelastic deformation of contacting bodies. The latter results in the dependence of energy losses due to plastic flow on the history of the sample deformation. In such a situation, the energy of adhesion if calculated based on the thermodynamic parameters of contacting bodies can be considered as a lower bound of the work spent on the separation of bodies.

A complex physical picture accompanying the adhesive contact breaking and the difficulties in measuring the total energy consumption required for the whole process of phase separation motivate using the force characteristics of the adhesive contact, such as adhesive strength. Thus, ice adhesion strength can be considered as the main practical measure of the proneness of the surfaces of the material to the accretion of solid water precipitations. Studies on materials that reduce ice and snow adhesion are continuously expanding. It was found that the magnitude of ice adhesion strength is affected by the environmental temperature and humidity, ice formation route and its properties, ice sample size and experimental method of adhesion strength measurement. During recent years, many promising coatings were designed, from which the ice can be more easily shed under wind, gravity, or vibration [8-17]. In this paper, we will discuss the mechanisms of adhesion of ice to surfaces and briefly review the factors promoting the reduction of ice adhesion strength and discuss some recent methods for the measurement of ice adhesion strength to the icephobic engineering materials. The role of the quasi-liquid layer (QLL) in the ice adhesion strength and its temperature dependence will be elucidated.

\section{Work of Adhesion of a Liquid Aqueous Medium to Solid Surfaces}

We will begin our consideration from the analysis of the adhesion of liquid water droplets to solid surfaces. As mentioned above, the contact angle formed by a liquid meniscus with a solid surface is a measure of intermolecular interactions, surface forces, and, respectively, the adhesion of liquid to a solid. This simplifies adhesion interpretation because in many cases instead of the investigation of different types of surface forces for various three-phase systems, the measurement and the analysis of the contact angle behavior allows getting information required to control the adhesion.

To quantitatively characterize the adhesion of a liquid to a smooth solid, the work of adhesion, $W_{s l}^{s m o o t h}$, calculated on the basis of Young-Dupre Equation (1) gives a straightforward and physically clear approach. If one considers the practical work of adhesion [18], associated with a liquid meniscus removal normally to a solid surface, it is necessary to use the receding contact angle [1,2], because such thermodynamic process is accompanied by the deformation of a liquid meniscus when liquid front recedes leaving a continuous or patched liquid film behind the receding meniscus:

$$
W_{s l}^{s m o o t h}=\sigma_{l v}\left(1+\cos \theta_{s l(r)}^{s m o o t h}\right)
$$


where $\sigma_{l v}$ is the surface tension of a given liquid (more exactly, liquid-vapor interfacial tension), which is determined by the properties of a liquid and the surface forces of the first kind $[19,20]$ acting in the vicinity of a liquid/vapor interface, $\cos \theta_{s l}^{\text {smooth }} r$ is a receding contact angle formed by a meniscus of liquid with the smooth solid, adhesion to which is under consideration. This contact angle is affected by the properties of all three phases being in contact: the liquid, the solid, and the vapor and thus, are defined by the surface forces of the second kind $[19,20]$ or, in the other words, by the isotherm of the disjoining pressure. The Young contact angle for any three-phase system can be calculated on the basis of the disjoining pressure isotherms using the Derjaguin-Frumkin approach [19-21]. In general, for the analysis of the wettability of solids by aqueous liquids the van der Waals, ion-electrostatic, steric, hydrophobic, and hydrophilic structural forces should be accounted for [19-22]. To decrease the adhesion energy for a given liquid, one needs to modify the properties of a three-phase system in a way, leading to an increase in the contact angle.

The latter can be achieved in different ways. For example, one could change the composition of aqueous phase or $\mathrm{pH}$ to attain different signs of surface charges for water/air and water/solid interfaces with a decrease in the magnitude of surface charges. This will affect the electrostatic forces. The deposition of the low polarizability nanosized layer on top of solid results in some water repelling properties associated with the van der Waals and/or hydrophobic forces.

The roughening of the solid surface can be considered as one of the most effective ways to vary the work of adhesion. Thus, for the rough solid surface with the roughness factor $r$ equal to the ratio of actual surface area to the apparent one (Figure 1a), for the solid wetted in Wenzel mode the Young-Dupre equation for the work of adhesion, $W_{s l}^{W}$, normalized to the unit of apparent contact area, could be easily derived in the form:

$$
W_{s l}^{\mathrm{W}}=r \cdot \sigma_{l v}\left(1+\cos \theta_{s l(r)}^{s m o o t h}\right)=r \cdot W_{s l}^{s m o o t h}
$$

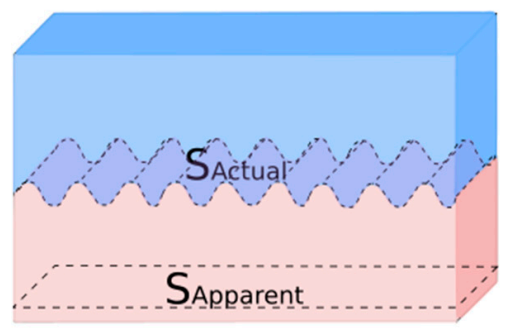

Wenzel mode

(a)

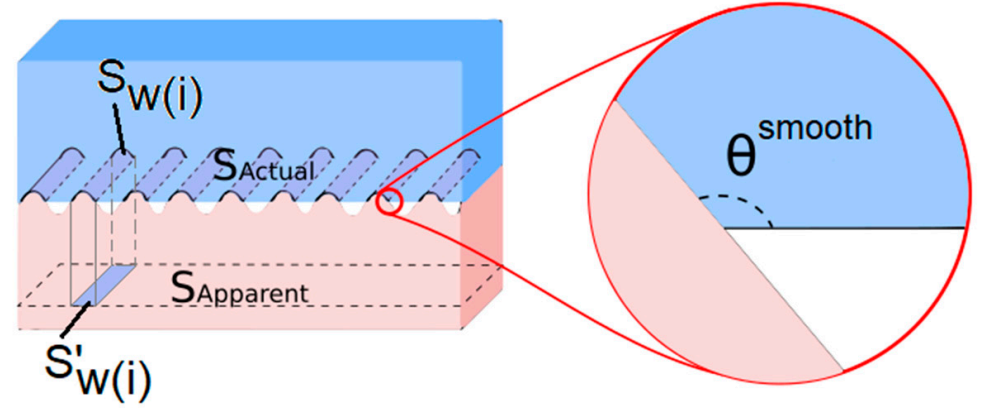

Cassie-Baxter mode

(b)

(c)

Figure 1. Schematic representation of wetting of the rough substrate in different modes explaining some definitions. (a) In a homogeneous (Wenzel) wetting mode the liquid fills all the depressions (grooves) of the surface relief; the roughness factor is determined as the ratio of actual and apparent (projected) surface areas; (b) In the heterogeneous (Cassie-Baxter) wetting mode air inclusions remain between the liquid and the solid surfaces; two factors are essential to describe this wetting mode, the fraction of apparent area directly contacting with the liquid, and the roughness factor of that wetted area; (c) At the equilibrium, the local contact angle at the groove wall is equal to one defined by the Young equation for the flat smooth surface with the same chemistry.

Since the roughness factor $r \geq 1$, roughening of the surface, according to Equation (2), will result in an increase in the work of adhesion for both hydrophilic and hydrophobic surfaces, in comparison to the smooth surfaces with the same chemical composition. However, simultaneous roughening and hydrophobization of the surface may lead to either increase or decrease of work of adhesion with 
respect to a bare smooth surface, depending on the parameters of the modified surface. When the rough superhydrophobic solid is wetted in Cassie-Baxter mode, the Young-Dupre equation for the work of adhesion, $W_{s l}^{C B}$, of liquid and solid phases (Figure $1 \mathrm{~b}$ ), normalized to the unit of apparent contact area, modifies to:

$$
W_{s l}^{C B}=r_{f} \cdot f \cdot \sigma_{l v}\left(1+\cos \theta_{s l(r)}^{s m o o t h}\right)=r_{f} \cdot f \cdot W_{s l}^{\text {smooth }}
$$

where $f$ is the projected fraction of solid area directly contacting with the liquid (wetted area fraction, $f=\left(\sum_{i} S_{w i}^{\prime}\right) / S_{\text {apparent }}$, see Figure $\left.1 \mathrm{~b}\right) ; r_{f}$ is the roughness factor for the wetted area $\left(r_{f}=\left(\sum_{i} S_{w i}\right) /\left(\sum_{i} S_{w i}^{\prime}\right)\right) ; \theta_{s l(r)}^{s m o o t h}$ has a sense of a local receding contact angle in the three-phase contact zone (Figure 1c).

The analysis of Equation (3) indicates that upon receding of a liquid meniscus for the solids wetted in the heterogeneous (Cassie-Baxter) mode with a low fraction of the wetted area $f<1 \%$, the work of adhesion will be significantly less than the work defined for the smooth surface with the same chemical composition of the surface layer. The situation becomes clearer for the substrates, having the posts textures with flat tops. For such textures, the liquid does not penetrate between the posts, wetted area roughness factor $r_{f}=1$, and Equation (3) reduces to the form:

$$
W_{s l}^{C B}=f \cdot W_{s l}^{s m o o t h}
$$

which indicates that for some particular textures and the apparent receding contact angle $\theta_{s l(r)}^{C B}>174^{\circ}$ for such surface, the work of adhesion may be less than $1 \%$ of $W_{s l}^{\text {smooth }}$.

Temperature sensitivity of the work of adhesion of a liquid to solid surfaces results from the variation of the contact angle and the surface tension with the temperature. One of the major conditions for the icephobic behavior of a given surface is the preservation of the low value of work of adhesion even for the deeply supercooled state of aqueous liquids. Experimental data indicate that the environmental temperature decrease to low negative values is accompanied by some increase in work of adhesion (Figure 2) due to both the contact angle deterioration and the rise of liquid surface tension $[9,13,23]$.

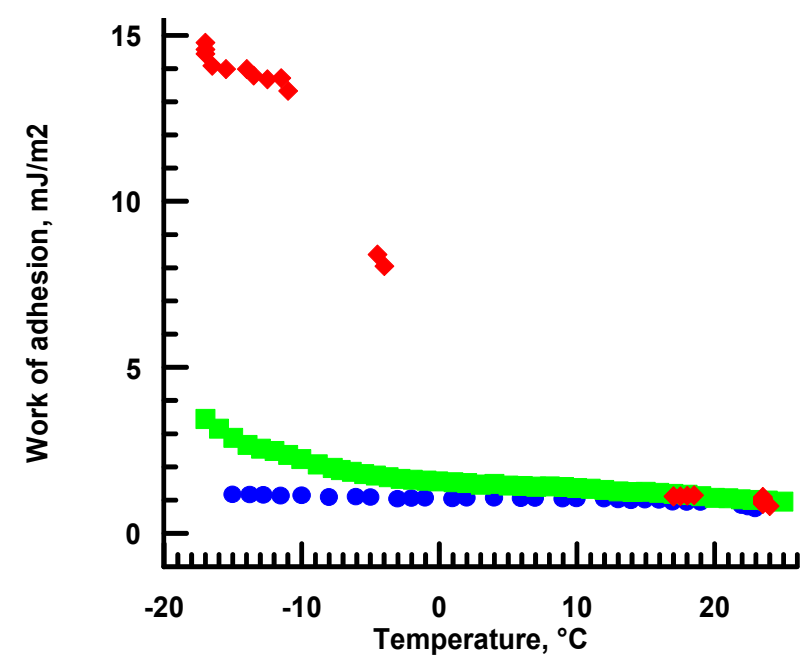

Figure 2. Practical work of adhesion for liquid aqueous droplet (supercooled liquid at negative temperatures): red diamonds—for water on superhydrophobic PDMS [13]; green squares—for $0.5 \mathrm{M}$ $\mathrm{NaCl}$ aqueous solutions on superhydrophobic aluminum substrates [23]; blue circles-for water on superhydrophobic aluminum substrates [23]. 


\section{The Mechanisms of Adhesion of Solid Water Medium to the Surfaces}

The establishing and the failure of contact of two solids are typically taking place in the irreversible processes, and the time required for the relaxation of the solid-solid contact to the equilibrium state is many orders of magnitude higher than that for the contact of a liquid to a solid. That is why the contact angle formed by one solid on top of the other, unlike a liquid-on-solid case, cannot be considered as a reliable and unambiguous characteristic of intermolecular and surface forces. In this situation, various thermodynamic and mechanical properties of contacting phases, as well as parameters of the geometrical contact, should be studied to interpret and control the adhesion.

As mentioned above, both chemical and physical mechanisms play a role in the magnitude of adhesion of solid water medium to solid surfaces of different nature. A chemical bonding mechanism, including the chemical reactions and hydrogen bonding between the hydroxyls grafted to the solid surface and hydroxyls of ice, is strongly sensitive to the chemical nature of adhesive solid. Chemical or hydrogen bonding mechanisms can provide adhesive energy, which is determined by the number of chemically active centers on a solid surface and the Gibbs free energy for chemical reaction or hydrogen bonding on each center. The effect of covalent and hydrogen bonding on the enhancement of adhesion of ice and water to solids was detected experimentally [24-26] in agreement with theoretical expectations.

The van der Waals contribution to the adhesion energy of ice to adhesive solid is determined by the difference between the frequency-dependent dielectric permittivity, $\varepsilon(i \xi)$, of contacting phases $[27,28]$. The numeric calculations of dielectric responses $\varepsilon(i \xi)$ for ice, water, and different solids, performed in [29], indicate very high dielectric contrast between the ice and different metals, and much lower contrast between the plastics or polymers and ice. This allows concluding significantly higher van der Waals contribution to the adhesion between the metal and ice in comparison to the adhesion of ice to polymeric materials. It is worth noting that although the van der Waals forces are long-ranged, one can easily manipulate the van der Waals contribution to the adhesion energy between the ice and solid by deposition of an intermediate nanosized solid layer. For example, the monolayer of a dielectric with low dielectric permittivity, say hydrocarbon, adsorbed onto the metal surface will significantly decrease the van der Waals contribution to the adhesion energy between the ice and the metal.

As for the electrostatic contribution to the adhesion energy of ice to solids, it may be related to different mechanisms. One of the first considerations of such forces was given in [30]. The main idea of the study [30] is related to the polarization of a solid substrate by the surface charges in ice, resulted in initiating the image charge interactions. As discussed in [30], such charges in ice originate from the capture of protonic charge carriers upon water crystallization. The indication of such charging comes from both the experiment and the theory [31-35]. The energy of image charges interactions is defined by the ice surface charge density and the dielectric permittivity of the solid substrate. This contribution to the adhesion energy is expected to be the highest for the ice/metal interface and the lowest for the ice/low dielectric permittivity solids, like nonpolar polymers. However, this image charge induced electrostatic interaction may be significantly reduced due to the formation of the liquid-like or premelted water layer between the ice and the substrate. Dissociation of water leads to the formation of the double electric layers at all confining interfaces, such as the ice/water, water/solid, or water/air. For example, the water/air interface charging at neutral $\mathrm{pH}$ is mainly defined by hydroxyls adsorption [35]. The charge of the ice/water interface originates from the dissociation of dangling $\mathrm{OH}$ bonds, ion adsorption, and ion uptake in the ice crystal [36]. Charging of ice/water and water/solid interfaces, which was discussed in the literature [36-44], on the one hand, induces strong ion-electrostatic interactions between the opposite boundaries of the premelted (quasi-liquid) water layer, on the other hand, provides the screening of the image charge interactions between the charge careers in ice and the image charges in the substrate. Since the presence of a quasi-liquid layer (QLL) of the aqueous phase inside the thin pores or on the ice surface has a great implication for the control of ice adhesion to the different surfaces, we will consider it's nature and impact on the adhesion energy in more detail. 


\section{Quasi-Liquid Layer at Solid/Ice Interfaces}

The first discussions of the properties of a liquid layer appearing on the surface of free ice at a temperature slightly less than the water triple point belong to works of Faraday [45] and Thomson [46]. As discussed in our earlier studies [47,48], the structure of a liquid layer at phase boundaries and in spatially confined systems changes when affected by a field of surface forces acting near the interface. This, in turn, changes the density and the components of the pressure tensor in the pre-surface layer, eventually resulting in a shift of the triple point, $T$, for this layer. Depending on the character of changes in the parameters specified above, $T$ can shift either towards lower temperatures, which results in pre-melting as is the case for ice, or towards higher temperatures to ensure pre-solidification, as with medium-chain hydrocarbons [49,50]. The value of shift of the triple point $T$ for free ice surface layer is quantitatively dependent on the ratio of ice/vapor and water/vapor surface energies and the number density of water molecules in ice or water surface layer [47]. For the aqueous layer sandwiched between the solid and ice, the ratio of ice/solid and water/solid surface energies comes into play. According to the theoretical analysis, the increase in the range of action of surface forces, which is expected in the aqueous phase in the vicinity of a hydrophobic solid [51], leads to the thicker pre-melted layer. The temperature dependence of the thickness of the pre-melted layer is directly ruled by the range and the strength of surface forces $[47,48]$.

Alternative theoretical approaches, based on the analysis of the excess van der Waals energy of system containing ice with the premelted film lead to the contradictive conclusions regarding the existence and stability of such a liquid film, depending on the particular spectrum of the dielectric permittivity for water used for the calculations [29,52,53]. Nevertheless, the authors of these approaches consented on the importance of accounting for the ion-electrostatic interactions between the charged ice/vapor or water/vapor interfaces, in addition to the contribution of the van der Waals interactions [29,44].

Another approach associates the QLL with a surface layer, characterized by orders of magnitude increased concentration of ice defects, such as dangling $\mathrm{OH}$ bonds and ion defects [54]. The nonuniformity of the distribution of defects in the ice surface layer normally to the interface and the step-wise increase in their concentration upon increasing the ice temperature from absolute zero to the triple point $T$ causes the first-order phase transition from ice to QLL well below $T$.

Although experimental and numerical studies have confirmed the existence of the liquid-like layer atop of free surface of the ice or sandwiched between bulk ice and solids of different chemical nature, its molecular origins and physical properties are still actively debated.

We are not going to dwell on the detailed results of studies of a premelted layer on the ice/vapor interface. Instead, we would like to refer to the excellent reviews on free ice premelting presented in $[55,56]$. Here we will briefly consider a QLL at the substrate/ice interface, which explicitly affects the adhesion of ice to solids. A wide range of modern methods, such as X-ray diffraction [57,58] and reflectivity [59], NMR measurements [60], total internal reflection (TIR) Raman spectroscopy [60], neutron diffraction [61], differential scanning calorimetry [62-64], thermoporometry [65], dilatometry [66], vibrational sum frequency generation $[60,67,68]$ has been used to study the properties of nonfrozen layer separating ice and substrates. For such investigations, the systems with both flat and concaved geometry of the substrate surface were used.

The onset temperature of premelting was studied for the substrates with different wettability. A great number of experimental data was collected for ice/silicas interfaces for flat amorphous $\mathrm{SiO}_{2}$ plates [59,67,68], thin quartz capillaries [66], porous silica particles [60], and cylindrical pores in SBA15 and MCM-41 (two classes of silica mesoporous materials with hexagonally arranged pores) $[62-65,69,70]$. QLL separating silica and ice in mesoporous silica with the pore sizes of $2-4 \mathrm{~nm}$ was detected at temperatures as low as $-80^{\circ} \mathrm{C}[69,70]$ and the thickness of such layers was estimated in the range of $0.35-0.54 \mathrm{~nm}$. However, this series of experiments did not address the influence of the temperature on the thickness of QLL. 
The temperature dependence of the QLL thickness was estimated for porous silica particles imbibed with $\mathrm{D}_{2} \mathrm{O}$ using ${ }^{2} \mathrm{H}$ NMR measurements as a function of temperature [60] (Figure 3). It was found that the onset temperature was approximately $-25^{\circ} \mathrm{C}$ with the QLL thickness of the order of 1 monolayer. At the highest explored temperature of $1.5^{\circ} \mathrm{C}$ below the $\mathrm{D}_{2} \mathrm{O}$ melting point, the NMR-derived QLL thickness was around $1.5 \mathrm{~nm}$. Larger thicknesses (Figure 3) and onset temperature $\left(-17^{\circ} \mathrm{C}\right)$ were reported in [59], where using an X-ray reflectivity data, the QLL thicknesses versus temperatures were measured for single crystal ice in contact with flat amorphous silica layer. The data obtained in [59] and [60] well correlate with each other and demonstrate significantly less negative onset temperature than that detected in mesoporous silica $[62-65,69,70]$. The fact of the notably higher thickness of QLL at onset temperatures for mesoporous silica with the pore sizes of a few $\mathrm{nm}$ is evidently related to the effect of the overlapping of the surface force fields induced by the opposite boundaries of the nanopores in SBA15 and MCM-41. Such an overlapping was shown to be quite significant for nonpolar liquids [71] and is expected to be much more important for highly polar liquids like water. It is worth noting that the $\mathrm{SiO}_{2}$ substrate is hydrophilic and in the case of a surfactant-free surface has a contact angle close to zero.

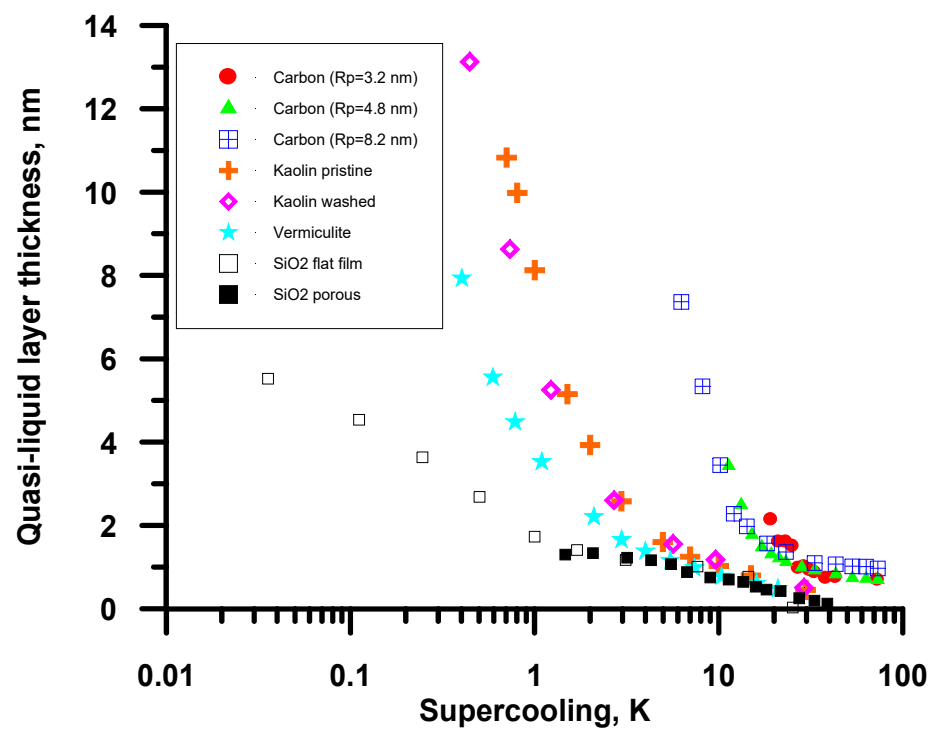

Figure 3. Thicknesses of a quasi-liquid layer on the ice surface in contact with different substrates as a function of supercooling with respect to bulk freezing temperature. Experimental data for porous carbon with different pore radii [58] and for kaolin (pristine and washed) and vermiculite clays [57] were estimated from high energy X-ray diffraction; for flat amorphous $\mathrm{SiO}_{2}$ film on $\mathrm{Si}$ block by the X-ray reflectivity measurements [59]; and for porous $\mathrm{SiO}_{2}$ particles imbibed with $\mathrm{D}_{2} \mathrm{O}$ by NMR studies [60].

Another example of hydrophilic surfaces inducing the formation of QLL between the ice and the clays was considered in [57]. Two types of clays were studied in contact with ice: vermiculite, which gets charged in an aqueous medium, and two uncharged kaolin clays, pristine and washed. The contact angle for clays was not presented in [57]; at the same time, the literature data indicated the hydrophilic nature of the surface of the above materials with the contact angle around $30^{\circ}$ [72]. High energy X-ray diffraction studies of QLL at kaolin/ice and vermiculite/ice interfaces [57] indicated some thickness increase with respect to those measured in the vicinity of $\mathrm{SiO}_{2}$ interfaces $[59,60]$ for a low degree of supercooling. At the same time, the available collection of data reported on QLL thickness versus temperature for hydrophilic materials well correlate with each other for deep supercooling (Figure 3).

The impact of more hydrophobic substrates on the QLL thickness was studied in cylindrical pores of ordered mesoporous crystalline carbons [58] and on the basal plane of exfoliated graphite [61]. For both hydrophobic substrates, the QLL was detected for the temperatures far below bulk water freezing 
temperature. Thus, the onset temperature of QLL for graphite was close to $-30^{\circ} \mathrm{C}$. Ordered mesoporous carbons with the pore radii of 3.2, 4.8 and $8.2 \mathrm{~nm}$ induced the QLL appearance, whose thicknesses estimated using X-ray diffraction were $0.68,0.71$, and $0.98 \mathrm{~nm}$, respectively, at $-73^{\circ} \mathrm{C}$ [58]. These values slightly exceed the values presented above for hydrophilic substrates (Figure 3). The comparative experiments on the freezing temperature of water in MCM- 41 with the same pore diameter of $2.3 \mathrm{~nm}$, modified by either hydrophilic or hydrophobic chain surfactants [62] indicated that in the hydrophobic pore, the liquid state persisted down to $-67^{\circ} \mathrm{C}$, which was more than 10 degrees lower than that for the hydrophilic pore.

To summarize the experimental data presented in the literature, we can conclude the impact of the surface wettability on the thickness and onset temperature of the premelted water layer separating ice and substrate. The tendency which follows from the analysis is that QLL thickness gets larger at more hydrophobic solids, and freezing temperatures for QLL in the vicinity of hydrophobic materials are lower than the temperatures measured for QLL bounded by the hydrophilic materials. This tendency well correlates with the data, obtained for the ice/vapor interface [56] if one considers that air is the most hydrophobic substance.

Having in mind the ubiquitous presence of QLL on ice/vapor or ice/solid interfaces at moderate supercooling, it is reasonable to estimate the impact of such a premelted layer on the work of adhesion of ice to a solid substrate. The presence of such a liquid layer allows hypothesizing that the process of separation of ice/substrate contact can be reversibly performed through the liquid film. In such a case, the work of adhesion will give useful information about the energy costs of the phase separation process. Let us consider the process of an interfacial contact breaking as sketched in Figure 4. The initial state of the system corresponds to ice and solid separated by a thin premelted water film with the thickness $h_{0}$, characterized by the interfacial film energy $\sigma_{s w i}[19]$ :

$$
\sigma_{s w i}=\sigma_{s w}+\sigma_{w i}+\int_{h_{0}}^{\infty} \Pi(h) d h
$$

where indices $s, w, i$ at $\sigma$ correspond to a solid substrate, water, and ice phases, respectively; triple index swi denotes the solid/ice boundary with thin water film in between; $\int_{h_{0}}^{\infty} \Pi(h) d h$ is the excess energy of interaction of ice and solid through the liquid film; $\Pi(h)$ is the disjoining pressure isotherm.

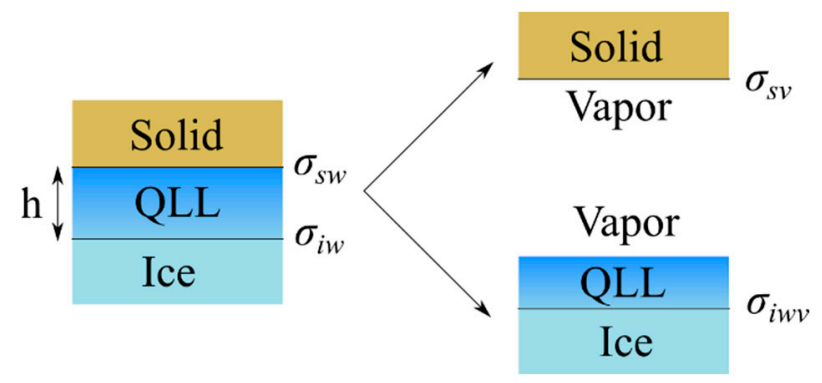

Figure 4. The scheme of the process for ice-solid contact separation in the presence of a quasi-liquid layer (QLL) on the ice surface.

For the final state of the system, the energy is equal to the sum of the interfacial solid/vapor energy $\sigma_{s v}$ and the interfacial ice/vapor energy $\sigma_{i w v}$, where ice is covered by a thin premelted layer.

Then, for the smooth solid/ice interface in the presence of QLL, the work of adhesion for the presented initial and final states can be calculated as:

$$
W_{s w i}=\sigma_{s v}+\sigma_{i w v}-\sigma_{s w}-\sigma_{w i}-\int_{h_{0}}^{\infty} \Pi(h) d h
$$


Using the Young-Dupre Equation (1) for the energy of adhesion of the water to smooth ice and of the water to smooth solid, one can immediately get:

$$
W_{s w i}=\sigma_{w v}\left(\cos \theta_{i w}+\cos \theta_{s w}\right)-\int_{h_{0}}^{\infty} \Pi(h) d h
$$

where $\theta_{i w}$ and $\theta_{s w}$ are the receding contact angles formed by water meniscus with either ice or solid surface, respectively.

Experimental studies $[52,73,74]$ on the wettability of ice with supercooled water indicate a notable spread in the values of $\theta_{i w}$ angle from $0^{\circ}$ to $12^{\circ}$. For the purposes of the analysis of the work of adhesion, the interval of possible contact angle values for water on ice justifies using the approximation $\cos \theta_{i w(r)} \approx 1$. Thus, we can write:

$$
W_{s w i} \approx \sigma_{w v}\left(1+\cos \theta_{s w}\right)-\int_{h_{0}}^{\infty} \Pi(h) d h=W_{s w}-\int_{h_{0}}^{\infty} \Pi(h) d h
$$

Equation (8) allows concluding that the work of adhesion of ice to smooth solid when the premelted layer is present in between, will exceed the work of adhesion of supercooled water to the same solid on the contribution of the excess free energy of interaction between the ice and solid across the premelted layer. As it was discussed above, different components of the disjoining pressure $[19,22]$ (and not only the van der Waals forces) will contribute to the excess energy of the water interlayer. The significant contribution of structural and the electrostatic components is additionally substantiated by the drastic, many orders of magnitude, increase in the viscosity of the nonfreezing layer, detected in the experiments [43,75]. The absence of data on the different components of the disjoining pressure in the water films confined by solid and ice does not allow performing rigorous quantitative calculations of the ice/solid adhesion energy. However, rough estimates of the integral in Equation (8) based on the Derjaguin-Frumkin theory of wetting indicate its negative sign. Typically, the magnitude of this integral is the higher, the smaller is the water interlayer thickness, and is expected to be of the order of ice/water interfacial energy. Overall, we can conclude that the adhesion energy of ice to solid exceeds the water/solid adhesion energy and is dependent on the solid wettability by both ice and water.

If the water layer between solid surface and ice is thick, the integral $\int_{h_{0}}^{\infty} \Pi(h) d h=0$, and for breaking the contact inside this thick layer, the work spent for ice-solid separation will be equal to the work of cohesion in water $W_{c}=2 \sigma_{w v}$.

Following an analysis presented above, one can expect a decrease in the adhesion strength of the ice/solid contact for negative temperatures close to the water triple point. Besides the lower work of breaking the adhesive contact for solid/QLL/ice interface compared to direct solid/ice contact, such a decrease is further promoted by less energy dissipation for heating and/or deforming the separated bodies. Studies of the contact failure for the ice/solid systems for both hydrophilic [60] and hydrophobic [2,15] solid surfaces, where the presence of QLL was experimentally confirmed, demonstrated adhesion strength reduction, affected by temperature and type of adhesion strength measurement. In shear measurements, depending on the thickness of the water layer between the ice and the solid surface, two mechanisms of ice/solid interaction with relative displacement were considered in the literature.

When the QLL thickness is small compared to the height of the surface texture, rather high local temperatures can develop during shearing. In such a case, mutual displacement is accompanied by the local melting of the ice ridges, leading to the lubrication action of QLL, as was shown in [76]. Having in mind, that the hydrophobization of surfaces with fluorinated long-chain surfactants, on the one hand, increase the contact angle and hence, as discussed above, the QLL thickness, and on the other hand, is characterized by improved tribological properties [77,78], one could expect lower values of shear adhesion strength for such hydrophobic solid surfaces. In the case when the thickness 
of the liquid layer is larger than the roughness size, ice shear is accompanied by the simultaneous flow of the liquid layer itself and with the viscous losses of the energy [75].

The positive impact of the nonfreezing liquid layer on the reduction of ice/solid adhesion strength, on the one hand, and the critical dependence of this strength on the temperature and the roughness, on the other hand, cause the development of the alternative approaches to decrease the adhesion strength. Two groups of the developing methods are based on the utilization of lubricating properties of the liquid layer located between the ice and the solid. The first one is related to using the slippery liquid infused porous surfaces (SLIPS) [14], where the thick layer of immiscible lubricant is impregnated into a textured solid to create a smooth liquid overlayer. The second direction is exploiting the formation of a hydrated water layer due to the film of hydrogel or hydrophilic polymers/brushes with a high affinity to water $[15,78]$ grafted onto the solid surface. We will not consider here the advantages and the problems of these approaches, related to SLIPS and some modifications of this method or hydration water layers, and address the readers to numerous recent reviews and papers in the field.

Another very prospective approach for the adhesion strength reduction is based on quite different principles and is associated with a drastic increase in the roughness of a hydrophobic solid, leading to establishing the heterogeneous wetting mode and the superhydrophobic state.

\section{Adhesion of Ice to Superhydrophobic Solids}

Recent studies have shown that the adhesion strength of ice to superhydrophobic coatings, characterized by low wetting hysteresis and receding contact angles exceeding $160^{\circ}$, can be considerably lower than that in the case of hydrophobic coatings $[9,13,14,79-82]$. To clarify further analysis, we should remind here that the wetting of the superhydrophobic surface occurs in a heterogeneous regime, that is, water partially contacts the solid surface and partially the air bubbles trapped inside the surface texture. To quantitatively characterize the adhesion for such surface, one needs to measure the actual adhesive strength for the studied sample and the actual adhesive contact area, which is determined by the product of the wetted area and the roughness factor of the wetted area. However, the measurement of the actual contact area is a challenging task and this surface area is typically unknown for the investigator. That is why to characterize the experimentally measured adhesive strength the apparent area is typically used. At the same time, as shown in the literature $[83,84]$ the actual area may be of the order of a few percent of the apparent area $(f \sim 0.01 \div 0.1)$. Here we would remind that for the hydrophobic surface the wetted area fraction $f=1$. Hence, the measured adhesion strength normalized on the apparent contact area for the superhydrophobic surfaces was expected to be 1 or 2 orders of magnitude less than that in the case of the hydrophobic surface with the same chemical composition of the surface layer.

However, the analysis of the experimentally measured values indicates that the adhesion strength for the superhydrophobic surfaces appears to be significantly higher than it was expected. Besides, in some cases the adhesion strength for the superhydrophobic surfaces with high contact angle and low wetting hysteresis shows to be even higher than that for a smooth hydrophobic substrate with the same chemical composition of the surface layer [85].

We believe that two main physical reasons may cause such phenomena. The first is a trivial one and is related to the non-uniform deposition of the hydrophobic layer inside the micro and nanotexture of the surface. As the thickness of the textured layer is typically exceeding $1 \mu \mathrm{m}$, the inappropriate deposition of the hydrophobic agent may cause a depletion of the latter in the inner areas of nano and micropores. Measuring of the initial receding and advancing angles for such surfaces does not allow revealing the nonuniformity of the surface energy, because the testing procedure typically takes several minutes and during this time, the liquid meniscus does not contact with the deeper areas of the textured layer. However, the problems with the superhydrophobic surface preparation and low stability of a hydrophobic agent layer can be visualized through the long-term immersion of coating in aqueous medium or prolonged contact with ice $[86,87]$. The deterioration of both the receding and the advancing contact angles due to desorption of hydrophobic molecules from the surface or 
due to capillary condensation of water/ice inside the pores with the defects in hydrophobic molecules coverage, in turn, causes the increase in the actual contact area between the ice and the surface. As a result, the adhesion strength might be higher than that characteristic of the smooth hydrophobic surface. To overcome the problem of enhanced adhesion strength associated with this reason, one needs to carefully test the superhydrophobic surface at room temperatures prior to starting icing experiments.

The second reason for enhanced ice adhesion strength follows from the universal phenomenon of the release of the latent heat of freezing and is inherent in the freezing process regardless of the substrate properties. Since the first stage of crystallization of a liquid meniscus contacting the substrate can be considered as a quasi-adiabatic process, the release of the heat of freezing results in some increase in the liquid temperature compared to the temperature of the ambient air and substrate. This phenomenon was detected experimentally [88-90] and leads to a short-term local increase in vapor pressure in the vicinity of the liquid meniscus. The local vapor supersaturation compared to the values characteristic of the substrate temperature, in turn, results in the desublimation or capillary condensation of supersaturated vapors on the substrate around the freezing droplet and inside the texture under the droplet [91]. In the case of desublimation, a notable increase in the ice/substrate contact area will take place. Interestingly that the stronger is the initial liquid supercooling, the higher will be vapor supersaturation. For example, for the liquid droplet supercooled before freezing till $-20{ }^{\circ} \mathrm{C}$, the temperature increase to nearly $0{ }^{\circ} \mathrm{C}$ due to the release of crystallization heat will cause temporal and local vapor supersaturation around $460 \%$, while crystallization of the droplet supercooled to $-5^{\circ} \mathrm{C}$ is accompanied by $\sim 140 \%$ of supersaturation.

Thus, the superhydrophobic substrates, which easily sustain a heterogeneous regime of wetting by supercooled water, may undergo a transition to the homogeneous regime even at not very significant supersaturations, resulting in the enlargement of wetted area. According to the experimental studies on the onset temperature for QLL (discussed above), it is reasonable to expect the formation of the frozen halo and meniscus around the droplet contact area on top of the substrate and within the microporous texture under the droplet. In nanotexture, water is expected to be present in a supercooled state. All the abovementioned processes lead to an increase in adhesion strength of ice to a solid due to both increased area of real contact and possible mechanical ice interlocking with overhanging elements of texture. However, it was shown in [91], that for superhydrophobic textures resistant to prolonged contact with ice and/or water condensed into nanopores, the ice desublimated into wider micropores and the frost halo around the frozen meniscus are being in a metastable state. This causes the spontaneous sublimation/evaporation of water from both the frost halo and the porous texture after the vapor pressure relaxation to saturated vapors. Although this process takes many hours, it promotes a decrease in the actual adhesion strength of the iced spot and can be successfully exploited in practice to facilitate the detachment of ice from superhydrophobic surfaces.

Thus, the analysis presented above allows concluding that, on the one hand, the small area of contact between a chemically stable and condensation resistant superhydrophobic surface and ice, due to the heterogeneous wetting regime, and the presence of QLL on the ice surface between ice and a substrate or inside the porous texture, on the other hand, facilitate ice removal from the surface. As it was shown by perennial outdoor experiments $[9,13]$, such superhydrophobic surfaces demonstrate spontaneous ice shedding under combined effects of gravity, wind force, or vibration.

The development of materials and coatings for outdoor applications requires immense laboratory studies of the chemical and mechanical resistance of fabricated materials, their adhesion to liquid and solid water at different environmental conditions. As it was shown in numerous recent critical reviews [92-94], the absolute values of ice adhesion depend on the peculiarities of methods of measurement and ice formation [81,92,93,95-97]. In the following section, we will briefly discuss the most general approaches to the measurement of ice adhesion to solids with the emphasis on the methods, which were not yet actively discussed in the literature and which allow studying complex shear stresses, mixed mode fracture and determining the temperature dependence of ice adhesion strength. 


\section{Adhesion Strength Measurements}

The methods for ice adhesion measurements can be classified in various ways [92-94,98]. Such classification accounts for the types of stresses, arising in the interfacial area and a three-phase contact zone upon loading. In general, four types of stresses promote solid/solid contact failure, such as tensile, shear, peel, and cleavage stresses. Although in some cases the role of all the above stresses which come into play for definite system geometry and contacting body properties is debatable $[92,94,98,99]$, most often two types, tensile and shear stresses, are considered in the literature, as responsible for ice/solid contact breaking. We did not set as our goal the detailed analysis of available measurement methods and address readers to the excellent recent reviews. Instead, we only intend here to briefly dwell on the application of these methods in some recent works, which expand our understanding of the nature of the adhesive contact of ice with modern engineering materials.

As it was stressed in the literature [79], the measurement of tensile adhesion strength, when a load is applied normally to ice/solid interface is not always correct, since, for high values of adhesion strength, the cohesive failure of ice is very likely (Figure 5a). At the same time, namely in the measurements of the tensile adhesion strength the wide variety of different ice samples, ice composition and ice faces become available for the analysis. The example of a device for realization of possibilities of measuring the adhesion to different ice faces and for natural ices from different regions, including Arctic zone ice, lake ices, etc. to flat plates is presented in Figure 5b.

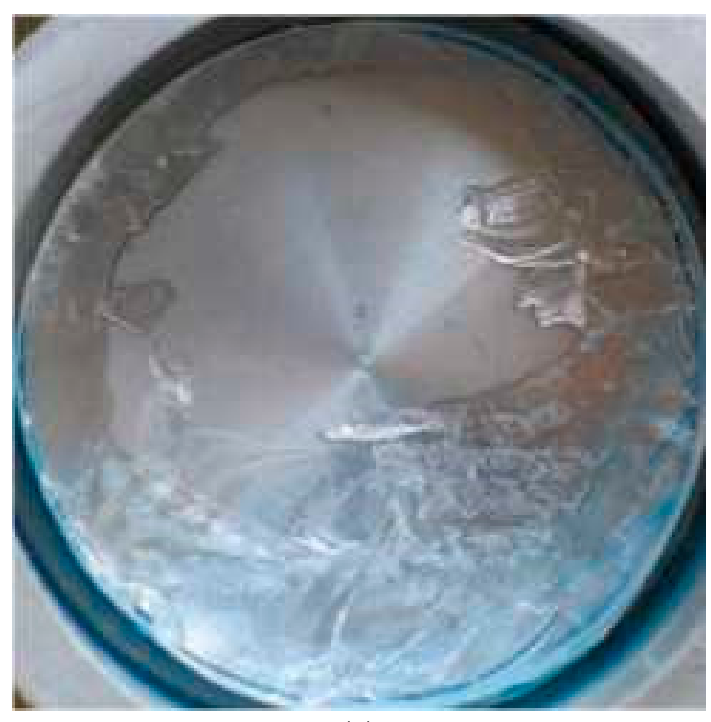

(a)

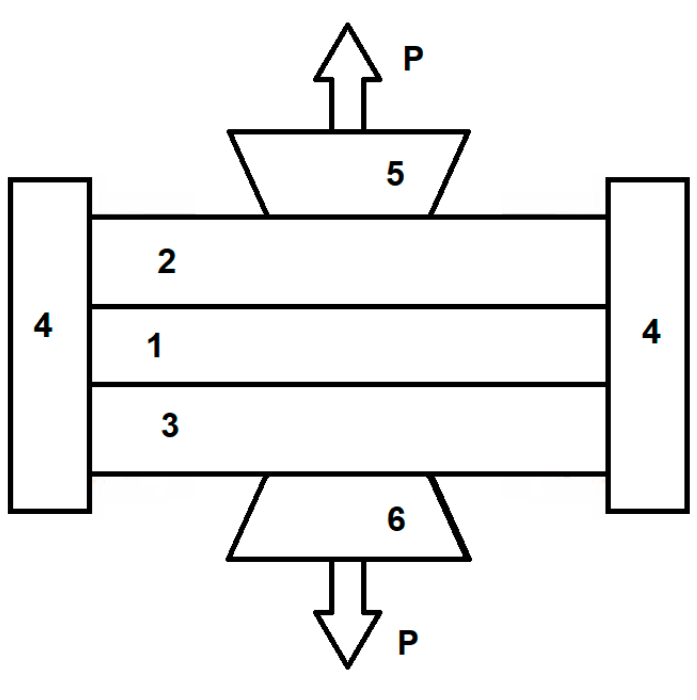

(b)

Figure 5. (a) A mixed adhesive-cohesive mode of fracture of ice-aluminum contact in tensile adhesion test; (b) Scheme of an experimental device for measuring the tensile adhesion strength of ice. 1-ice slab; 2 and 3-disks with reference or test surfaces; 4-guide sleeve; 5 and 6-clutches; P-applied tensile force.

The device consists of two coaxially arranged test disks 2 and 3 and an ice plate 1 frozen between them [100]. A thin fluoroplastic ring (not shown in the figure) is placed between the guide sleeve 4 and the disks 2 and 3 . The ratio of the diameter of the disks to the thickness of the sample is about 20, which corresponds to the creation of a uniform stress field inside the ice sample, provided that the ice is homogeneous. Besides, a large contact area allows minimization of the impact of different types of local stresses arising at the sample edges on the measured value of tensile adhesion strength. Test disks are connected to clutches 5 and 6 of the electromechanical universal testing machine which performs tensile mechanical tests. The whole device is placed within a thermostatic cooling chamber. In [100], for testing the tensile adhesion strength of ice to aluminum disks with various coatings, the slices of natural ices cut from the surface of the natural basin were used. Flat ice plates were brought into 
contact with aluminum disks at a temperature of about $0{ }^{\circ} \mathrm{C}$ and cooled to the test temperature at a rate of $3{ }^{\circ} \mathrm{C} / \mathrm{h}$, followed by equilibration for $2-16 \mathrm{~h}$. The testing machine applied tensile mechanical load $\mathrm{P}$ and determined its maximum value Pmax corresponding to the breaking of the contact between disks 2 and 3 through ice layer 1 . Visual inspection allowed discriminating between adhesive and cohesive fracture. In some cases, when the ice plate was frozen to the degreased or chemically etched hydrophilic surfaces of the aluminum disks, a mixed mode of fracture can be observed (Figure 5a). It is worth noting that literature data on ice tensile adhesion strength to the uncoated metal surfaces, measured for mixed mode fracture typically demonstrate wide scattering [95,101].

Another undoubted advantage of methods based on tensile adhesion strength measurement is the ability to test with high spatial resolution the ice adhesion to the surfaces with a wettability gradient. For this, it is convenient to use the approach developed in [102], where the tensile adhesion strength was measured for an individual small water droplet, suspended on a hydrophilized coil attached to the tensiometer system through the thin wire. The droplet freezing on the particular area of a cooled substrate followed by further detachment allowed measuring the tensile strength with the spatial resolution determined by the droplet contact area.

For the shear adhesion strength measurements, a wide scattering of the experimental data is related to poorly defined detaching stresses, as well. The latter are affected by the position and type of applied force, by the speed of loading, temperature, properties of ice, etc. Shear adhesion strength is determined, to a large extent, by the number of defects in the near-surface layer and the character of deformation in the contacting bodies. For various kinds of ices (glaze, rime, or mixed), the variation in the number of defects in the vicinity of interface and the differences in the degree of deformation under the same load result in very distinct values of shear adhesion strength. At the same time, it should be stressed that at very low temperatures when QLL is absent on the interface, one can conclude much easier shear and delamination of ice on the hydrophobic and the superhydrophobic surfaces based on the general physical analysis. This is the consequence of the Rehbinder's effect which correlates the occurrence, growth, and propagation of cracks with the value of the surface energy of the crack's walls. For cracks, which propagate strictly along with the interface during shearing, one of the crack's walls coincides with the hydrophobic surface, having low surface energy, thus facilitating the delamination. To decrease the impact of the nonuniformity of interfacial stresses and the defects in ice on the measured shear strength, the set-ups like one shown in Figure 6a can be used.

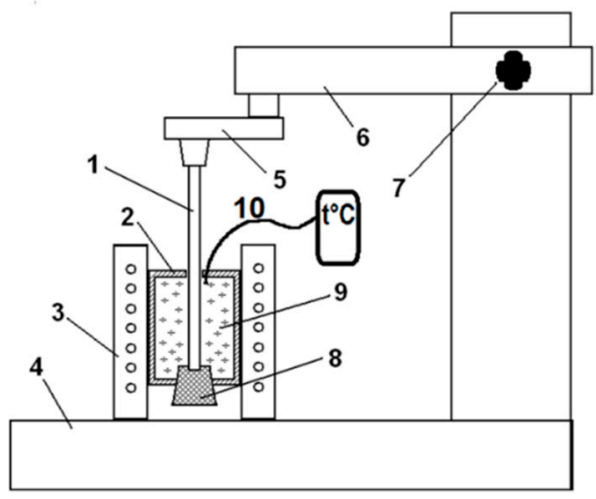

(a)

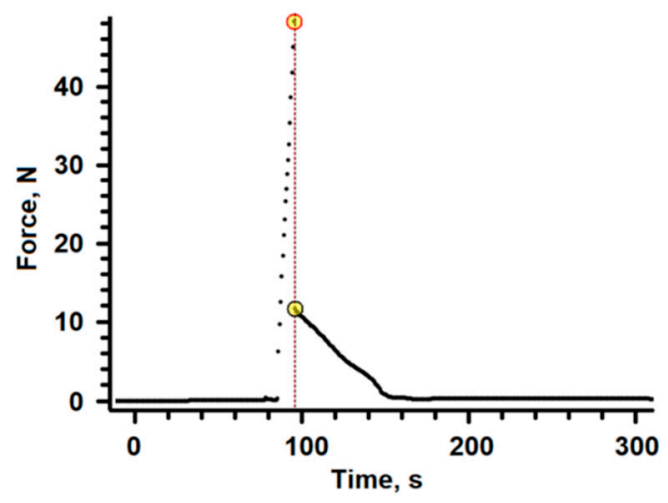

(b)

Figure 6. (a) Scheme of an experimental setup for measuring the shear adhesion strength of ice [103]. 1-test sample; 2-vessel with water/ice; 3-freezing device; 4-base; 5-force sensor; 6-movable lever;7-lever drive knob; 8-plug; 9-ice; 10-thermocouple; (b) Typical time dependence of force measured by the force sensor. Circles mark the maximum load force and the residual force after the failure of adhesion contact. Note that the non-zero residual force here results from the friction of an ice and the sample upon its movement in the iced bath after adhesion contact breaking. 
In this setup, the determination of adhesive strength was based on using the force sensor 5 to measure the axial force required to pull the cylindrical sample 1 out of the ice 9 formed in the vessel 2 cooled by the freezing device 3 . The force determined by the sensor 5 was recorded continuously using a computer program. The temperature of the sample was monitored using a thermocouple 10, the junction of which was in frozen water in the immediate vicinity of the sample. The plug 8 made from PTFE was used to protect the bottom end of sample 1 from contact with ice and thus to exclude the tensile strength contribution to the measured force. In the system with the described geometry, the surface shear stresses are distributed more evenly, without the participation of external torques and other types of stresses.

To ensure the thermal equilibration, the force measurements started not earlier than one hour after establishing the set temperature in the device. The value of the force $F$ required to pull the sample out of the ice was determined as the difference between the maximum load and the "residual" one, i.e. the load that remains after the failure of the adhesion contact (Figure 6b). The shear adhesion strength was then calculated as $F / S$, where the contact area $S$ was determined from the sample radius and the depth of its immersion in ice. This setup allows easily getting the temperature dependence of a shear adhesion strength and study the effects of resistance of the surface texture to cyclic adhesion contact failure [103]. Besides, it allows one to study the effects of ice creep, local melting of the ice ridges, and lubrication action of QLL on the value of shear adhesion strength (Figure 7).

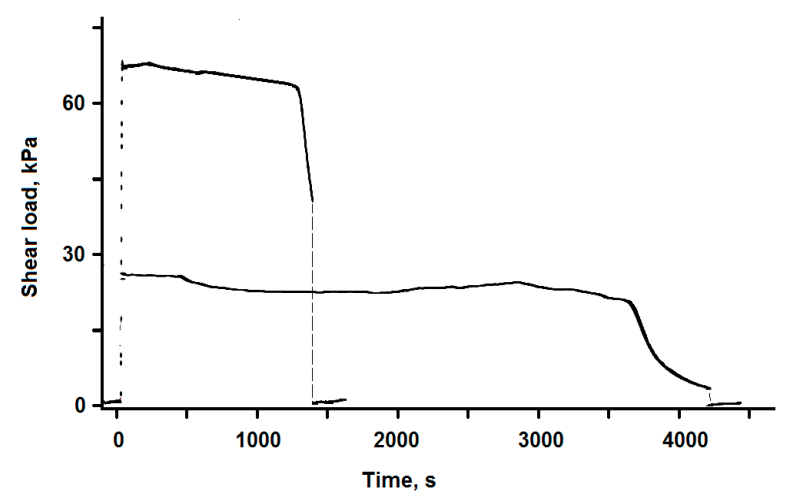

Figure 7. Dependence of shear stress measured by the loading force sensor on the time elapsed after the load is applied along the axis of an aluminum cylinder with a superhydrophobic surface, one of the ends of which is frozen in the ice at $-8^{\circ} \mathrm{C}$. Two dependences corresponding to different values of the initial load are presented [4].

The study on the failure of adhesive contact between a superhydrophobic coating on the aluminum wire and an ice sleeve under shear conditions $[4,103]$ has shown that even at very low prolonged shear stress, the sample slowly slides out of the ice sleeve due to successive sliding/melting processes (Figure 7). Seemingly, the same processes allow spontaneous shedding of snow and ice from the surface of superhydrophobic plates under weak wind load upon their exposure in winter environmental conditions [9].

Before completing this section, we would like to briefly discuss the possibility of studying complex shear stresses for ice deposited on engineering materials. Considering that for many practical applications of structural materials the conditions of ice adhesive strength at combined loads differ from the conditions corresponding to any single type of loading, it was important to develop an appropriate methodology and its hardware implementation for measuring ice adhesion to structural materials. The need for such tests arises, for example, when studying the adhesion of ice to current-carrying cables of overhead power lines. Cables have a complex curved surface formed by twisted layers of individual cable wires. As a result, under the action of a shear load along the cable axis, a torque also appears, i.e. a complex shear acts on the ice-cable surface (a combination of longitudinal and transverse shear). 
To address this aspect, a device shown in Figure 8, which allowed applying and studying a complex load combining axial and rotational forces was used [100]. In this device, the test sample 1 is clamped in two clutches 2 and 3. An ice sleeve 4 is formed around the sample with an aid of a split collar (not shown in the scheme). In the measurement run, the ice sleeve through the tapered bushing 5 is propped by the support bushing 6 , and the axial force $P$ is applied to the test sample through the clutch 2 and the thrust bearing 7 . To apply the axial load $P$, the electromechanical universal testing machine was used, in a compression mode. The rotational motion was transmitted to the ice ring through the clutch 2 by applying the torque $M$ created by a pair of forces $F_{1}$ in the plane orthogonal to the axis of the rod. Thus, complex shear stresses acted on the ice-rod contact interface due to a combination of the external axial force $P$ and the torque $M$.

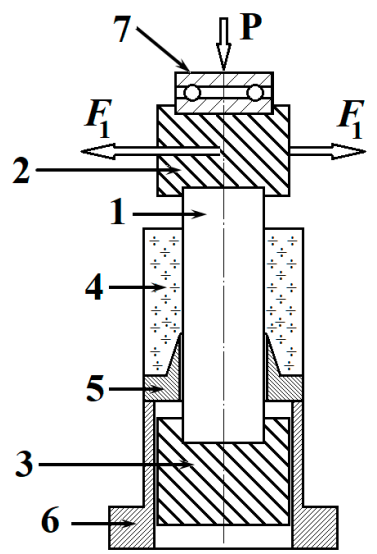

(a)

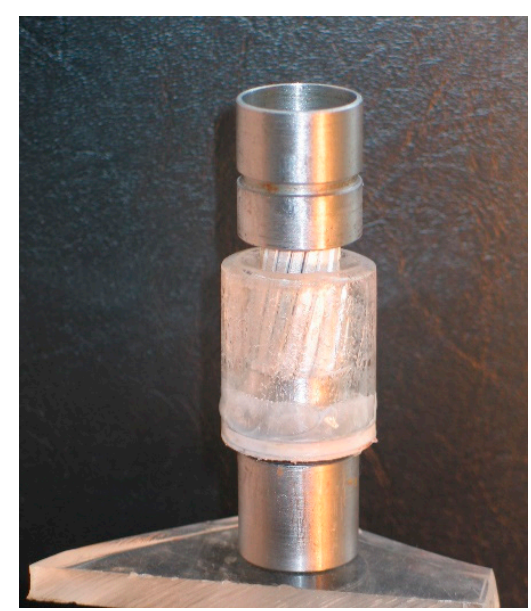

(b)

Figure 8. (a) Scheme of the device for testing ice adhesion to electric cables in conditions of combined shear [100]. 1—test sample; 2 and 3-clutches; 4-ice sleeve; 5-tapered bushing; 6—support bushing; 7-flat-thrust bearing; P-applied axial load; F1-F1 - pair of forces applying a torsion torque; (b) Photo of the ice sleeve on the electric cable clamped between two clutches.

The device allows one to experimentally check the effect of additional shear stresses on the adhesive strength of ice, i.e. to realize the conditions of a complex shear in an ice crust frozen on a cylindrical rod. The instant of the breaking of the adhesive contact is defined as the point of reaching the maximum angle of rotation, which occurs at the maximum of shear force, after which the ice crust glides along the aluminum wires of the cable. However, this boundary between the statics and dynamics of shear may be expressed not clearly/sharply enough; therefore, the acoustic emission method was applied [104]. The method of deformation acoustic emission makes it possible to clearly track the true moment at which the adhesive contact of ice frozen on the wire breaks and, consequently, a critical angle of rotation is reached. The latter in [103] was measured by the optical method (from the displacement of the laser beam reflected from the mirror, fixed on the cable sample).

Using the above described and other well-established methods, the values of tensile and shear adhesion strengths were obtained for numerous metal and polymeric surfaces. The analysis of this wide set of data allows concluding high variability of results obtained for the same type of substrates. That is why one of the most urgent tasks, repeatedly stressed in recent reviews is related to the standardization of experimental conditions, such as ice adhesion tests, types of accreted ice, environmental conditions, and surface parameters $[92,93,99]$. At the same time, already collected data allows discussing not only the adhesion strength itself, but its temperature dependence, which plays a major role in the design of materials aimed to be used in different outdoor applications. 


\section{Temperature Dependence of Ice Adhesion Strength to Different Surfaces and Coatings}

The impact of temperature on the adhesion strength is affected by a number of factors, such as the density and the distribution of surface and bulk stresses, the temperature dependence of surface forces, which crucially affect the contact angle and the thickness of the premelted aqueous layer or embedded slippery layer. Besides, the temperature of ice formation is a determining factor for the structure, density, and mechanical properties of the ice itself [105]. Hence, to estimate the ability of material for spontaneous or artificial ice shedding upon exploitation, the temperature dependence of the adhesion strength for the practically important temperature range is highly desirable. Numerous early data analyzed in $[92,106]$ gave somewhat contradictory results. However, in essence, most of the data obtained by the application of various methods for the adhesion strength measurement revealed a decrease in the ice adhesion strength with increasing of the temperature, approaching to the triple point. For example, the combined data for aluminum and steel, grouped by test category (cf. Figures 34 and 35 in [92], respectively) shows a trend of linear increase in adhesion strength with the temperature decrease. More recent results on shear adhesion strength obtained for the smooth hydrophilic surface of silica and smooth aluminum substrates coated with substituted n-alkyldimethylalkoxysilanes, both hydrogen bonding and non-hydrogen bonding, well agree with this trend $[60,107,108]$. For the superhydrophobic substrates obtained using different methods of texturing and using various hydrophobic molecules, the trends for the temperature dependence of glaze ice shear adhesion strength (Figure 9) well agree with each other. As for the values of ice adhesion strength to different superhydrophobic surfaces, presented in the literature, they, for example for the glaze ice at $-10{ }^{\circ} \mathrm{C}$, scatter in a wide range from $9 \mathrm{kPa}$ for textured Teflon [86], to $70 \mathrm{kPa}$ for fluorooxysilane on silicon rubber [13], and to $200 \mathrm{kPa}$ for anodized aluminum with a monolayer of fluorooxysilane [103].

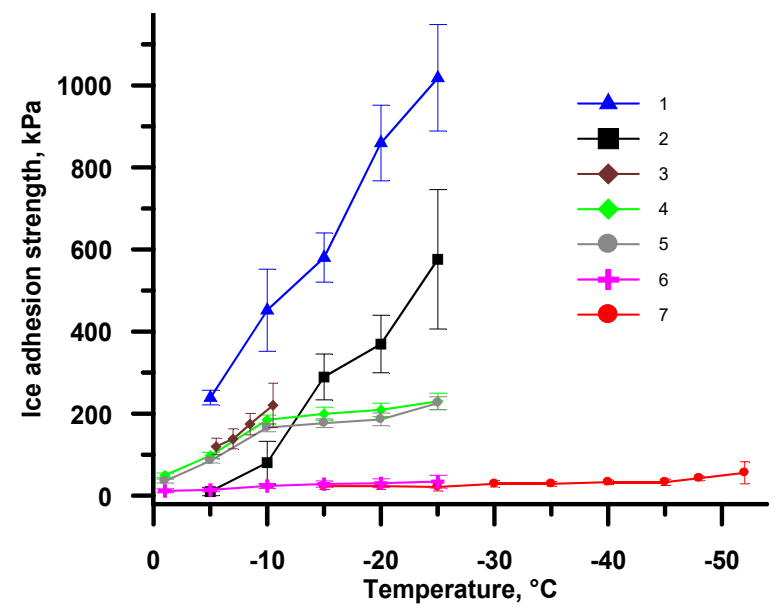

Figure 9. Temperature dependences of ice shear adhesion stress for different substrates: 1—ultra-smooth hydrophilic silica substrates $\left(\mathrm{R}_{\mathrm{a}}<0.4 \mathrm{~nm}\right)$ [60]; 2-silica plates coated with an adsorbed diblock copolymer layer [78]; 3-superhydrophobic aluminum rod [103]; 4, 5, 6-micro-nano structured aluminum surface modified by perfluorooctyltrichlorosilane, polydimethylsiloxane (PDMS), and silicon-oil-infused PDMS-modified surface, respectively [12]; 7-dimethylolpropionic acid-polyurethane composite coating with an aqueous lubricating layer [109].

Now let us consider the temperature dependence of ice adhesion strength for the systems, where the ice is separated from the solid by either embedded slippery oil film or by the thick hydration water layer. For the former case, the oily layer thickness will be defined by the peculiarities of the texture on solid. For the latter case, the stability and freezing of a layer of hydration water are ruled by the chemical or hydrogen bonding interactions with the functional groups of a hydrophilic polymer $[12,78,108-110]$. In both situations, the thickness of a liquid layer will be of the order or exceed the surface roughness. Here, two mechanisms have to be mentioned as responsible for the ice shearing force. The first one is 
related to the difference in capillary forces arising in the advancing and receding menisci of the liquid layer wetting the ice. However, this difference is two orders of magnitude lower than the forces needed to explain the experimentally measured shear stresses. The second mechanism is related to the transition from the external friction in the boundary lubrication regime to the mixed regime with the hydrodynamic component of friction. In such a regime, to explain the existence of initial shearing force which is measured in the experiments on shear adhesion strength, the hypothesis should be accepted about a viscous-plastic behavior of liquid in slippery oil film or in the hydration water layer. Such behavior is characterized by stress threshold for liquid flowing, first proposed in [75], and later developed by different authors [111-114]. In particular, a method [114], optimizing the transmission of the shear stress to the sample, which enabled a more complete probe of the mechanical response of liquids, revealed a solid-like property for thin films of liquid water and viscous alkanes. The behavior of experimentally measured weak temperature dependence of shear strength for ice contacted to superhydrophobic substrate impregnated by silicon oil [12] and for aqueous layer sandwiched between an ice and a solid substrate coated by polyurethane-based polymer [109] correlated with the hypothesis on the stress threshold for supercooled liquid flowing as a mechanism responsible for ice adhesion shear strength in the presence of a thick liquid interlayer. Indeed, the measured shear strength is of the order of tens of $\mathrm{kPa}$ and remains nearly constant (see Figure 9) in a wide range of temperatures of -1 to $-25^{\circ} \mathrm{C}$ for silicone oil and of -15 to $-50{ }^{\circ} \mathrm{C}$ for the aqueous lubricating layer. The experimentally detected increase in shear strength for lower temperatures corresponds to the liquid films thinning and the transition from the mixed to boundary lubrication friction regime.

\section{Conclusions and Outlook}

In this manuscript, we tracked how a change in the surface wettability is related to the adhesion of liquid water and ice to the surface. The presented analysis showed that the adhesion of water to solids is mainly defined by the wettability. In contrast, the analysis of the mechanisms of ice adhesion to the same surfaces allows concluding a more complex situation. The thickness of a quasi-liquid aqueous layer (QLL) spontaneously formed between the solid surface and ice under the influence of surface forces is one of the key factors determining the strength of adhesive contact and the work of ice adhesion to the solid surface. It was discussed that the hydrophobic nature of the surface layer of a solid promotes both a decrease in van der Waals forces and screening of the image charge contributions to the ice adhesion. Additionally, hydrophobicity of the surface shifts the onset temperature of QLL formation to lower temperatures; the thickness of QLL in the vicinity of hydrophobic surfaces shows to be higher than near the hydrophilic surfaces, resulting in a more wide temperature interval of the positive impact of QLL on the ice removal. Chemical structure of the hydrophobic molecules can further promote the ice shear from the surface through the specific tribological properties of, for example, fluorocompounds. For the chemically and mechanically stable superhydrophobic surfaces an additional factor facilitating the ice and snow shedding is related to a drastic decrease in a fraction of solid area actually contacting the water/ice. Besides, other factors affecting the ice adhesion strength to solid surfaces, which are beyond the scope of this brief review, were intensively discussed in the literature. For example, two competing mechanisms were identified contributing to the ice adhesion strength, such as mechanical interlocking of the ice within the surface features that enhances adhesion $[85,115,116]$, and formation of microcracks, responsible for the adhesion reduction [10,115,117].

However, despite some particular bright achievements, for example, for the superhydrophobic coatings [9], in general, the presented analysis indicates that the ice adhesion strength to solid surfaces is often higher than that necessary for spontaneous ice shedding. That is why additional approaches are still desirable to further decrease the adhesion strength between an ice and a solid. Here we briefly mentioned new strategies related to the utilization of lubrication properties of either thick oily slippery layer or the low-freezing hydration water layer. Although some attractive features of these two new approaches for the design of icephobic surfaces were already illustrated in the literature, plenty of studies should be performed before the general industrial applicability of these new coatings will 
be substantiated. The main points to be resolved for the hydrophilic polymer coatings on metals, promoting the formation of thick hydration water layers are related to the analysis of the resistance of such surfaces to atmospheric and electrolyte corrosion. Besides, the problem of spontaneous hydrophobization of such surfaces in contact with airborne organic contaminations remains unresolved. For oil slippery layer infused into surface polymeric or textured metal matrix, the key point still requiring a thorough analysis is the prolonged behavior of such coatings upon exposure to real open atmospheric conditions, characteristic of large industrial cities with dust, aggressive atmospheric gases and aqueous precipitation.

Funding: This research was funded by the Russian Foundation for Basic Research, grant number 19-29-13011, and by the Ministry of Science and Higher Education of the Russian Federation.

Conflicts of Interest: The authors declare no conflict of interest. The funders had no role in the design of the study; in the collection, analyses, or interpretation of data; in the writing of the manuscript, or in the decision to publish the results.

\section{References}

1. Gao, L.C.; McCarthy, T.J. Wetting $101^{\circ}$. Langmuir 2009, 25, 14105-14115. [CrossRef] [PubMed]

2. Meuler, A.J.; Smith, J.D.; Varanasi, K.K.; Mabry, J.M.; McKinley, G.H.; Cohen, R.E. Relationships between water wettability and ice adhesion. ACS Appl. Mater. Interfaces 2010, 2, 3100-3110. [CrossRef] [PubMed]

3. Voitchovsky, K.; Kuna, J.J.; Contera, S.A.; Tosatti, E.; Stellacci, F. Direct mapping of the solid-liquid adhesion energy with subnanometre resolution. Nat. Nanotechnol. 2010, 5, 401-405. [CrossRef] [PubMed]

4. Boinovich, L.B.; Emelyanenko, A.M. Anti-icing potential of superhydrophobic coatings. Mendeleev Commun. 2013, 23, 3-10. [CrossRef]

5. He, Z.; Vagenes, E.T.; Delabahan, C.; He, J.; Zhang, Z.L. Room temperature characteristics of polymer-based low ice adhesion surfaces. Sci. Rep. 2017, 7, 42181. [CrossRef]

6. Sun, Y.J.; Jiang, Y.H.; Choi, C.H.; Xie, G.Y.; Liu, Q.X.; Drelich, J.W. Direct measurements of adhesion forces of water droplets on smooth and patterned polymers. Surf. Innov. 2018, 6, 93-105. [CrossRef]

7. Derjaguin, B.V.; Krotova, N.A.; Smilga, V.P. Adhesion of Solids; Johnston, R.K., Translator; Consultants Bureau: New York, NY, USA, 1978. (In Russian)

8. Golovin, K.; Kobaku, S.P.R.; Lee, D.H.; DiLoreto, E.T.; Mabry, J.M.; Tuteja, A. Designing durable icephobic surfaces. Sci. Adv. 2016, 2, e1501496. [CrossRef]

9. Boinovich, L.B.; Emelyanenko, A.M.; Emelyanenko, K.A.; Modin, E.B. Modus operandi of protective and anti-icing mechanisms underlying the design of longstanding outdoor icephobic coatings. ACS Nano 2019, 13, 4335-4346. [CrossRef]

10. Golovin, K.; Dhyani, A.; Thouless, M.D.; Tuteja, A. Low-interfacial toughness materials for effective large-scale deicing. Science 2019, 364, 371-375. [CrossRef]

11. Coady, M.J.; Wood, M.; Wallace, G.Q.; Nielsen, K.E.; Kietzig, A.M.; Lagugne-Labarthet, F.; Ragogna, P.J. Icephobic behavior of UV-cured polymer networks incorporated into slippery lubricant-infused porous surfaces: Improving SLIPS durability. ACS Appl. Mater. Interfaces 2018, 10, 2890-2896. [CrossRef]

12. Barthwal, S.; Lee, B.; Lim, S.-H. Fabrication of robust and durable slippery anti-icing coating on textured superhydrophobic aluminum surfaces with infused silicone oil. Appl. Surf. Sci. 2019, 496, 143677. [CrossRef]

13. Emelyanenko, A.M.; Boinovich, L.B.; Bezdomnikov, A.A.; Chulkova, E.V.; Emelyanenko, K.A. Reinforced superhydrophobic coating on silicone rubber for longstanding anti-icing performance in severe conditions. ACS Appl. Mater. Interfaces 2017, 9, 24210-24219. [CrossRef] [PubMed]

14. Kreder, M.J.; Alvarenga, J.; Kim, P.; Aizenberg, J. Design of anti-icing surfaces: Smooth, textured or slippery? Nat. Rev. Mater. 2016, 1, 15003. [CrossRef]

15. Chen, D.; Gelenter, M.D.; Hong, M.; Cohen, R.E.; McKinley, G.H. Icephobic surfaces induced by interfacial non-frozen water. ACS Appl. Mater. Interfaces 2017, 9, 4202-4214. [CrossRef]

16. He, Z.; Wu, C.; Hua, M.; Wu, S.; Wu, D.; Zhu, X.; Wang, J.; He, X. Bioinspired multifunctional anti-icing hydrogel. Matter 2020, 2, 723-734. [CrossRef]

17. Liu, F.T.; Wang, Z.K.; Pan, Q.M. Intelligent icephobic surface toward self-deicing capability. ACS Sustain. Chem. Eng. 2020, 8, 792-799. [CrossRef] 
18. Mittal, K. Adhesion measurement: Recent progress, unsolved problems, and prospects. In Adhesion Measurement of Thin Films, Thick Films and Bulk Coatings; Mittal, K., Ed.; ASTM International: West Conshohocken, PA, USA, 1978; pp. 5-7. [CrossRef]

19. Derjaguin, B.V.; Churaev, N.V.; Muller, V.M. Surface Forces; Consultants Bureau: New York, NY, USA; London, UK, 1987. [CrossRef]

20. Boinovich, L.; Emelyanenko, A. Wetting and surface forces. Adv. Colloid Interface Sci. 2011, 165, 60-69. [CrossRef]

21. Churaev, N.V.; Sobolev, V.D. Prediction of contact angles on the basis of the Frumkin-Derjaguin approach. Adv. Colloid Interface Sci. 1995, 61, 1-16. [CrossRef]

22. Boinovich, L.B. Long-range surface forces and their role in the progress of nanotechnology. Russ. Chem. Rev. 2007, 76, 471-488. [CrossRef]

23. Chulkova, E.V.; Emelyanenko, A.M.; Emelyanenko, K.A.; Boinovich, L.B. Parameters of supercooled droplets of water and aqueous solutions of chlorides of alkali metals in the temperature range of +25 to $-19^{\circ} \mathrm{C}$. Russ. J. Phys. Chem. A 2020, 94, 564-569. [CrossRef]

24. Sonwalkar, N.; Sunder, S.S.; Sharma, S.K. Ice solid adhesion analysis using low-temperature Raman microprobe shear apparatus. Appl. Spectrosc. 1993, 47, 1585-1593. [CrossRef]

25. Petrenko, V.; Peng, S. Reduction of ice adhesion to metal by using self-assembling monolayers (SAMs). Can. J. Phys. 2003, 81, 387-393. [CrossRef]

26. Matsumoto, K.; Tsubaki, D.; Sekine, K.; Kubota, H.; Minamiya, K.; Yamanaka, S. Influences of number of hydroxyl groups and cooling solid surface temperature on ice adhesion force. Int. J. Refrig. 2017, 75, 322-330. [CrossRef]

27. Lifshitz, E.M. The theory of molecular attractive forces between solids. Sov. Phys. JETP 1956, 2, 73-83. [CrossRef]

28. Dzyaloshinskii, I.E.; Lifshitz, E.M.; Pitaevskii, L.P. The general theory of van der Waals forces. Adv. Phys. 1961, 10, 165-209. [CrossRef]

29. Wilen, L.A.; Wettlaufer, J.S.; Elbaum, M.; Schick, M. Dispersion-force effects in interfacial premelting of ice. Phys. Rev. B 1995, 52, 12426-12433. [CrossRef]

30. Ryzhkin, I.A.; Petrenko, V.F. Physical mechanisms responsible for ice adhesion. J. Phys. Chem. B 1997, 101, 6267-6270. [CrossRef]

31. Ryzhkin, I.A.; Petrenko, V.F. Proton ordering in ice at an ice-metal interface. J. Exp. Theor. Phys. 2005, 101, 317-321. [CrossRef]

32. Takahasi, T. Electric surface potential of growing ice crystals. J. Atmos. Sci. 1970, 27, 453-462. [CrossRef]

33. Sugimoto, T.; Aiga, N.; Otsuki, Y.; Watanabe, K.; Matsumoto, Y. Emergent high-Tc ferroelectric ordering of strongly correlated and frustrated protons in a heteroepitaxial ice film. Nat. Phys. 2016, 12, 1063-1068. [CrossRef]

34. Ryzhkin, I.A. Thermodynamics of Ice. Not Obeying the Rules. Nat. Phys. 2016, 12, 996-997. [CrossRef]

35. Beattie, J.K.; Djerdjev, A.M.; Warr, G.G. The surface of neat water is basic. Faraday Discuss. 2009, 141, 31-39. [CrossRef] [PubMed]

36. Inagawa, A.; Harada, M.; Okada, T. Charging of the ice/solution interface by deprotonation of dangling bonds, ion adsorption, and ion uptake in an ice crystal as revealed by zeta potential determination. J. Phys. Chem. C 2019, 123, 6062-6069. [CrossRef]

37. Kallay, N.; Cakara, D. Reversible charging of the ice-water interface: 1. Measurement of the surface potential. J. Colloid Interface Sci. 2000, 232, 81-85. [CrossRef]

38. Kallay, N.; Preočanin, T.; Kovačević, D.; Lützenkirchen, J.; Chibowski, E. Electrostatic potentials at solid/liquid interfaces. Croat. Chem. Acta 2010, 83, 357-370.

39. Cop, A.; Kallay, N. Surface potential of ice in aqueous electrolyte solutions. In Trends in Colloid and Interface Science XVII. Progress in Colloid and Polymer Science; Springer: Berlin/Heidelberg, Germany, 2004; Volume 126, pp. 83-85. [CrossRef]

40. Lee, J.; Rick, S.W. Characterizing charge transfer at water ice interfaces. J. Phys. Chem. Lett. 2012, 3, 3199-3203. [CrossRef]

41. Bryk, T.; Haymet, A.D.J. Profiles of electrostatic potential across the water-vapor, ice-vapor and ice-water interfaces. Condens. Matter Phys. 2016, 19, 13607. [CrossRef] 
42. Lyklema, J. Fundamentals of Interface and Colloid Science: Solid-Liquid Interfaces; Elsevier: Amsterdam, The Netherlands, 2005; Volume 2.

43. Lecadre, F.; Kasuya, M.; Harano, A.; Kanno, Y.; Kurihara, K. Low-temperature surface forces apparatus to determine the interactions between ice and silica surfaces. Langmuir 2018, 34, 11311-11315. [CrossRef]

44. Thiyam, P.; Fiedler, J.; Buhmann, S.Y.; Persson, C.; Brevik, I.; Bostrom, M.; Parsons, D.F. Ice particles sink below the water surface due to a balance of salt, van der Waals, and buoyancy forces. J. Phys. Chem. C 2018, 122, 15311-15317. [CrossRef]

45. Faraday, M. Royal Institution Discourse, June 7, 1850. In Experimental Researches in Chemistry and Physics; Taylor and Francis: New York, NY, USA, 1991.

46. Thomson, J., III. Note on Professor Faraday's recent experiments on "regelation". Proc. R. Soc. Lond. 1862, 11, 198-204. [CrossRef]

47. Boinovich, L.; Emelyanenko, A. To the problem of first order phase transition at the fluid-fluid interface. Colloids Surf. A 2007, 300, 321-326. [CrossRef]

48. Boinovich, L.; Emelyanenko, A. Shift of triple point in confined systems with curved interfaces. Mol. Phys. 2009, 107, 1745-1753. [CrossRef]

49. Sloutskin, E.; Bain, C.; Ocko, B.M.; Deutsch, M. Surface freezing of chain molecules at the liquid-liquid and liquid-air interfaces. Faraday Discuss 2005, 129, 339-352. [CrossRef] [PubMed]

50. Gang, O.; Wu, X.Z.; Ocko, B.M.; Sirota, E.B.; Sinha, S.K.; Deutsch, M. Surface freezing in chain molecules: II. Neat and hydrated alcohols. Phys. Rev. E 1998, 58, 6086. [CrossRef]

51. Boinovich, L.B.; Emelyanenko, A.M. Forces due to dynamic structure in thin liquid films. Adv. Colloid Interface Sci. 2002, 96, 37-58. [CrossRef]

52. Elbaum, M.; Schick, M. Application of the theory of dispersion forces to the surface melting of ice. Phys. Rev. Lett. 1991, 66, 1713-1716. [CrossRef]

53. Fiedler, J.; Boström, M.; Persson, C.; Brevik, I.H.; Corkery, R.W.; Buhmann, S.Y.; Parsons, D.F. Full-spectrum high resolution modeling of the dielectric function of water. J. Phys. Chem. B 2020, 124, 3103-3113. [CrossRef]

54. Ryzhkin, I.A.; Ryzhkin, M.I.; Sinitsyn, V.V.; Klyuev, A.V. Model of a surface liquid-like layer of ice. JETP Lett. 2017, 106, 760-764. [CrossRef]

55. Dash, J.G.; Fu, H.; Wettlaufer, J.S. The premelting of ice and its environmental consequences. Rep. Prog. Phys. 1995, 58, 115-167. [CrossRef]

56. Slater, B.; Michaelides, A. Surface premelting of water ice. Nat. Rev. Chem. 2019, 3, 172-188. [CrossRef]

57. Li, H.; Bier, M.; Mars, J.; Weiss, H.; Dippel, A.C.; Gutowski, O.; Honkimäki, V.; Mezger, M. Interfacial premelting of ice in nano composite materials. Phys. Chem. Chem. Phys. 2019, 21, 3734-3741. [CrossRef] [PubMed]

58. Morishige, K. Influence of pore wall hydrophobicity on freezing and melting of confined water. J. Phys. Chem. C 2018, 122, 5013-5019. [CrossRef]

59. Engemann, S.; Reichert, H.; Dosch, H.; Bilgram, J.; Honkimaki, V.; Snigirev, A. Interfacial melting of ice in contact with $\mathrm{SiO}_{2}$. Phys. Rev. Lett. 2004, 92, 205701. [CrossRef] [PubMed]

60. Liljeblad, J.F.D.; Furó, I.; Tyrode, E.C. The premolten layer of ice next to a hydrophilic solid surface: Correlating adhesion with molecular properties. Phys. Chem. Chem. Phys. 2017, 19, 305-317. [CrossRef]

61. Gay, J.M.; Suzanne, J.; Dash, J.G.; Fu, H. Premelting of ice in exfoliated graphite; a neutron diffraction study. J. Cryst. Growth 1992, 125, 33-41. [CrossRef]

62. Deschamps, J.; Audonnet, F.; Brodie-Linder, N.; Schoeffel, M.; Alba-Simionesco, C. A thermodynamic limit of the melting/freezing processes of water under strongly hydrophobic nanoscopic confinement. Phys. Chem. Chem. Phys. 2010, 12, 1440-1443. [CrossRef]

63. Schreiber, A.; Ketelsen, I.; Findenegg, G.H. Melting and freezing of water in ordered mesoporous silica materials. Phys. Chem. Chem. Phys. 2001,3, 1185-1195. [CrossRef]

64. Findenegg, G.H.; Jaehnert, S.; Akcakaryiran, D.; Schreiber, A. Freezing and melting of water confined in silica nanopores. ChemPhysChem 2008, 9, 2651-2659. [CrossRef]

65. Endo, A.; Yamamoto, T.; Inagi, Y.; Iwakabe, K.; Ohmori, T. Characterization of nonfreezable pore water in mesoporous silica by thermoporometry. J. Phys. Chem. C 2008, 112, 9034-9039. [CrossRef]

66. Bardasov, S.A.; Sobolev, V.D.; Churaev, N.V. Dependence of the thickness of nonfreezing water interlayers on the external-pressure. Colloid J. 1992, 54, 160-166. 
67. Wei, X.; Miranda, P.B.; Zhang, C.; Shen, Y.R. Sum-frequency spectroscopic studies of ice interfaces. Phys. Rev. B 2002, 66, 085401. [CrossRef]

68. Yamaguchi, S.; Suzuki, Y.; Nojima, Y.; Otosu, T. Perspective on sum frequency generation spectroscopy of ice surfaces and interfaces. Chem. Phys. 2019, 522, 199-210. [CrossRef]

69. Schmidt, R.; Hansen, E.W.; Stoecker, M.; Akporiaye, D.; Ellestad, O.H. Pore size determination of MCM-41 mesoporous materials by means of 1H NMR spectroscopy, N2 adsorption, and HREM. A preliminary study. J. Am. Chem. Soc. 1995, 117, 4049-4056. [CrossRef]

70. Hansen, E.W.; Stoecker, M.; Schmidt, R. Low-temperature phase transition of water confined in mesopores probed by NMR. Influence on pore size distribution. J. Phys. Chem. 1996, 100, 2195-2200. [CrossRef]

71. Boinovich, L.B. Boundary layers and surface forces in pure non-aqueous liquids. Curr. Opin. Colloid Interface Sci. 2019, 44, 85-93. [CrossRef]

72. Kholodov, V.A.; Milanovskiy, E.Y.; Konstantinov, A.I.; Tyugai, Z.N.; Yaroslavtseva, N.V.; Perminova, I.V. Irreversible sorption of humic substances causes a decrease in wettability of clay surfaces as measured by a sessile drop contact angle method. J. Soils Sediments 2018, 18, 1327-1334. [CrossRef]

73. Knight, C.A. Experiments on the contact angle of water on ice. Philos. Mag. 1971, 23, 153-165. [CrossRef]

74. Thiévenaz, V.; Josserand, C.; Séon, T. Retraction and freezing of a water film on ice. Phys. Rev. Fluids 2020, 5, 041601. [CrossRef]

75. Barer, S.S.; Churaev, N.V.; Derjaguin, B.V.; Kiseleva, O.A.; Sobolev, V.D. Viscosity of non-freezing thin interlayers between the surfaces of ice and quartz. J. Colloid Interface Sci. 1980, 74, 173-180. [CrossRef]

76. Budnevich, S.S.; Derjaguin, B.V. On the slip of solids on ice. Prog. Surf. Sci. 1994, 45, 262-276. [CrossRef]

77. Marczak, J.; Kargol, M.; Psarski, M.; Celichowski, G. Modification of epoxy resin, silicon and glass surfaces with alkyl- or fluoroalkylsilanes for hydrophobic properties. Appl. Surf. Sci. 2016, 380, 91-100. [CrossRef]

78. Heydari, G.; Tyrode, E.; Visnevskij, C.; Makuska, R.; Claesson, P.M. Temperature-dependent deicing properties of electrostatically anchored branched brush layers of poly(ethylene oxide). Langmuir 2016, 32, 4194-4202. [CrossRef]

79. Janjua, Z.A.; Turnbull, B.; Choy, K.-L.; Pandis, C.; Liu, J.; Hou, X.; Choi, K.-S. Performance and durability tests of smart icephobic coatings to reduce ice adhesion. Appl. Surf. Sci. 2017, 407, 555-564. [CrossRef]

80. Nguyen, T.-B.; Park, S.; Lim, H. Effects of morphology parameters on anti-icing performance in superhydrophobic surfaces. Appl. Surf. Sci. 2018, 435, 585-591. [CrossRef]

81. Idriss, H.; Guselnikova, O.; Postnikov, P.; Kolska, Z.; Hausild, P.; Cech, J.; Lyutakov, O.; Svorcik, V. Versatile and scalable icephobization of airspace composite by surface morphology and chemistry tuning. ACS Appl. Polym. Mater. 2020, 2, 977-986. [CrossRef]

82. Vazirinasab, E.; Maghsoudi, K.; Jafari, R.; Momen, G. A comparative study of the icephobic and self-cleaning properties of teflon materials having different surface morphologies. J. Mater. Process. Technol. 2020, 276, 116415. [CrossRef]

83. Boinovich, L.B.; Emelyanenko, A.M. The analysis of the parameters of three phase coexistence in the course of long term contact between a superhydrophobic surface and an aqueous medium. Chem. Lett. 2012, 41, 1241-1243. [CrossRef]

84. Boinovich, L.B.; Emelyanenko, A.M. The behaviour of fluoro- and hydrocarbon surfactants used for fabrication of superhydrophobic coatings at solid/water interface. Colloids Surf. A 2015, 481, 167-175. [CrossRef]

85. Chen, J.; Liu, J.; He, M.; Li, K.; Cui, D.; Zhang, Q.; Zeng, X.; Zhang, Y.; Wang, J.; Song, Y. Superhydrophobic surfaces cannot reduce ice adhesion. Appl. Phys. Lett. 2012, 101, 111603. [CrossRef]

86. Kulinich, S.A.; Honda, M.; Zhu, A.L.; Rozhin, A.G.; Du, X.W. The icephobic performance of alkyl-grafted aluminum surfaces. Soft Matter 2015, 11, 856-861. [CrossRef]

87. Boinovich, L.B.; Emelyanenko, A.M.; Pashinin, A.S. The analysis of long term durability of superhydrophobic properties under continuous contact with water. ACS Appl. Mater. Interfaces 2010, 2, 1754-1758. [CrossRef] [PubMed]

88. Jung, S.; Tiwari, M.K.; Poulikakos, D. Frost halos from supercooled water droplets. Proc. Natl. Acad. Sci. USA 2012, 109, 16073-16078. [CrossRef] [PubMed]

89. Alizadeh, A.; Yamada, M.; Li, R.; Shang, W.; Otta, S.; Zhong, S.; Ge, L.; Dhinojwala, A.; Conway, K.R.; Bahadur, V.; et al. Dynamics of ice nucleation on water repellent surfaces. Langmuir 2012, 28, 3180-3186. [CrossRef] [PubMed] 
90. Yin, L.; Xia, Q.; Xue, J.; Yang, S.; Wang, Q.; Chen, Q. In situ investigation of ice formation on surfaces with representative wettability. Appl. Surf. Sci. 2010, 256, 6764-6769. [CrossRef]

91. Boinovich, L.B.; Emelyanenko, A.M. Role of water vapor desublimation in the adhesion of an iced droplet to a superhydrophobic surface. Langmuir 2014, 30, 12596-12601. [CrossRef]

92. Work, A.; Lian, Y. A critical review of the measurement of ice adhesion to solid substrates. Prog. Aerosp. Sci. 2018, 98, 1-26. [CrossRef]

93. Rønneberg, S.; Zhuo, Y.; Laforte, C.; He, J.; Zhang, Z. Interlaboratory study of ice adhesion using different techniques. Coatings 2019, 9, 678. [CrossRef]

94. Schulz, M.; Sinapius, M. Evaluation of Different Ice Adhesion Tests for Mechanical Deicing Systems; SAE Technical Paper 2015-01-2135; SAE International: Warrendale, PA, USA, 2015. [CrossRef]

95. Tetteh, E.; Loth, E. Reducing static and impact ice adhesion with a self-lubricating icephobic coating (SLIC). Coatings 2020, 10, 262. [CrossRef]

96. Vercillo, V.; Tonnicchia, S.; Romano, J.-M.; García-Girón, A.; Aguilar-Morales, A.I.; Alamri, S.; Dimov, S.S.; Kunze, T.; Lasagni, A.F.; Bonaccurso, E. Design rules for laser-treated icephobic metallic, surfaces for aeronautic applications. Adv. Funct. Mater. 2020, 30, 1910268. [CrossRef]

97. Tarquini, S.; Antonini, C.; Amirfazli, A.; Marengo, M.; Palacios, J. Investigation of ice shedding properties of superhydrophobic coatings on helicopter blades. Cold Reg. Sci. Technol. 2014, 100, 50-58. [CrossRef]

98. Kasaai, M.R.; Farzaneh, M. A critical review of evaluation methods of ice adhesion strength on the surface of materials. In 23rd International Conference on Offshore Mechanics and Arctic Engineering; ASME: Vancouver, BC, Canada, 2004; Volume 3, pp. 919-926. [CrossRef]

99. Rønneberg, S.; He, J.; Zhang, Z. The need for standards in low ice adhesion surface research: A critical review. J. Adhes. Sci. Technol. 2020, 34, 319-347. [CrossRef]

100. Gol'dshtein, R.V.; Epifanov, V.P. To the measurement of ice adhesion to other materials. Bull. Perm State Tech. Univ. Mech. 2011, 2, 28-41. (In Russian)

101. Yeong, Y.H.; Milionis, A.; Loth, E.; Sokhey, J.; Lambourne, A. Atmospheric ice adhesion on water-repellent coatings: Wetting and surface topology effects. Langmuir 2015, 31, 13107-13116. [CrossRef] [PubMed]

102. Psarski, M.; Pawlak, D.; Grobelny, J.; Celichowski, G. Relationships between surface chemistry, nanotopography, wettability and ice adhesion in epoxy and SU-8 modified with fluoroalkylsilanes from the vapor phase. Appl. Surf. Sci. 2019, 479, 489-498. [CrossRef]

103. Boinovich, L.B.; Zhevnenko, S.N.; Emel'yanenko, A.M.; Gol'dshtein, R.V.; Epifanov, V.P. Adhesive strength of the contact of ice with a superhydrophobic coating. Dokl. Chem. 2013, 448, 71-75. [CrossRef]

104. Epifanov, V.P.; Glasovskiy, A.F. Acoustic parameters as indicators of peculiarities of ice movement in glaciers. Kriosfera Zemli (Earth Cryosphere) 2010, 14, 42-55. Available online: https://izdatgeo.ru/pdf/krio/2010-4/42.pdf (accessed on 26 May 2020). (In Russian).

105. Petrovic, J. Review mechanical properties of ice and snow. J. Mater. Sci. 2003, 38, 1-6. [CrossRef]

106. Work, A.H., Jr. The Measurement of the Adhesion of Glaze Ice. Electronic Theses and Dissertations Paper 2987. Ph.D. Thesis, University of Louisville, Louisville, KY, USA, 2018. [CrossRef]

107. Smith, J.G.; Whol, C.J.; Kreeger, R.E.; Palacios, J.; Hernandez, M. Effects of Hydrogen Bonding and Molecular Chain Flexibility of Substituted n-Alkyldimethylsilanes on Impact Ice Adhesion Shear Strength; NASA Technical Memorandum NASA/TM-2020-220567; NASA Langley Research Center: Hampton, VA, USA, 2020. Available online: https://ntrs.nasa.gov/archive/nasa/casi.ntrs.nasa.gov/20200002031.pdf (accessed on 26 May 2020).

108. Höhne, S.; Hoch, C.; Böhm, C.; Winkler, R.; Bittrich, E.; Uhlmann, P. A new measuring system for the determination of the ice adhesion strength on smooth surfaces. Langmuir 2020, 36, 4465-4476. [CrossRef]

109. Dou, R.; Chen, J.; Zhang, Y.; Wang, X.; Cui, D.; Song, Y.; Jiang, L.; Wang, J. Anti-icing coating with an aqueous lubricating layer. ACS Appl. Mater. Interfaces 2014, 6, 6998-7003. [CrossRef]

110. Chen, J.; Dou, R.; Cui, D.; Zhang, Q.; Zhang, Y.; Xu, F.; Zhou, X.; Wang, J.; Song, Y.; Jiang, L. Robust prototypical anti-icing coatings with a self-lubricating liquid water layer between ice and substrate. ACS Appl. Mater. Interfaces 2013, 5, 4026-4030. [CrossRef]

111. Derjaguin, B.V.; Bazaron, U.B.; Zandanova, K.T.; Budaev, O.R. The complex shear modulus of polymeric and small-molecule liquids. Polymer 1989, 30, 97-103. [CrossRef]

112. Derjaguin, B.V.; Bazaron, U.B.; Lamazhapova, K.D.; Tsidypov, B.D. Shear elasticity of low-viscosity liquids at low frequencies. Phys. Rev. A 1990, 42, 2255. [CrossRef] [PubMed] 
113. Badmaev, B.; Dembelova, T.; Damdinov, B.; Makarova, D.; Budaev, O. Influence of surface wettability on the accuracy of measurement of fluid shear modulus. Colloids Surf. A 2011, 383, 90-94. [CrossRef]

114. Noirez, L.; Baroni, P. Identification of a low-frequency elastic behaviour in liquid water. J. Phys.: Condens. Matter 2012, 24, 372101. [CrossRef]

115. Ling, E.J.Y.; Uong, V.; Renault-Crispo, J.S.; Kietzig, A.M.; Servio, P. Reducing ice adhesion on nonsmooth metallic surfaces: Wettability and topography effects. ACS Appl. Mater. Interfaces 2016, 8, 8789-8800. [CrossRef]

116. Menini, R.; Farzaneh, M. Advanced Icephobic Coatings. J. Adhes. Sci. Technol. 2011, 25, 971-992. [CrossRef]

117. Nosonovsky, M.; Hejazi, V. Why superhydrophobic surfaces are not always icephobic. ACS Nano 2012, 6, 8488-8491. [CrossRef]

(C) 2020 by the authors. Licensee MDPI, Basel, Switzerland. This article is an open access article distributed under the terms and conditions of the Creative Commons Attribution (CC BY) license (http://creativecommons.org/licenses/by/4.0/). 